\title{
Computational fluid dynamic (CFD) of vertical-axis wind turbine: mesh and time-step sensitivity study
}

\author{
S. Ashwindran. N*, A. A. Azizuddin, A. N. Oumer \\ ${ }^{1}$ Faculty of Mechanical Engineering, Universiti Malaysia Pahang, \\ 26600 Pekan, Pahang, Malaysia \\ *Email: azizuddin.ump.edu.my \\ Phone: +6094246231; Fax: +6094246222
}

\begin{abstract}
This paper presents mesh and time-step dependence study of newly designed drag type vertical axis wind turbine. Ansys FLUENT a commercially available CFD solver was used to perform CFD numerical study on the drag type wind turbine. In computational analysis, $2 D$ models was simulated under unsteady flow fields using SST $k-\omega$ to achieve stabilized numerical convergence. The model was analyzed at static and dynamic mode, where sliding mesh technique was used to analyze the turbine in dynamic mode. Three main parameters were taken under careful consideration: mesh resolution, turbulence model and time-step. Dimensionless quantity such as moment and drag was used in mesh sensitivity study for both static and sliding mesh. A small discrepancy in results of $2 D$ sliding mesh result at different time-step and mesh resolution was observed. The generated results showed good agreement between fine and medium mesh with trivial dissimilarities in the initial initialization. In timestep dependency study for static mesh, $d t=0.0002$ time-step size was chosen for economical computational cost.
\end{abstract}

Keywords: Vertical axis wind turbine; CFD; time-step; wind energy; sliding mesh

\section{INTRODUCTION}

Vertical axis wind turbine (VAWT) is categorized into two types, lift and drag type. Savonius wind turbine is a drag type, which have low rotation speed with high torque capabilities [1]. S-shaped Savonius wind turbine is not efficient in harvesting energy, therefore design improvement was made to ensure the design are able to adapt to low wind speed conditions [2]. Darrieus wind turbine is characterized as lift type wind turbine, which uses aerofoil shaped blade design to generate lift, which creates high rotation speed at low torque. According to Betz's limit, wind turbine efficiency of any design can't exceed 59.3\% [3]. The concept of designing a wind turbine system requires an in-depth knowledge on unsteady aerodynamics and principles of fluid mechanics. There is no restriction on the principal of designing a wind turbine system but understanding the mechanics of aerodynamics and aeroelasticity would provide a platform to design a functional wind turbine system. 


\section{Past CFD Numerical Analysis}

In transient CFD simulation, selecting the appropriate turbulent model is an essential element to generate a reliable and accurate result. Researcher has to have a throughout understanding on unsteady aerodynamic, turbulent algorithm and governing equations available in the respective CFD software. Since the aim of this paper is to study the unsteady response of the newly designed vertical axis wind turbine, hence, literature review study on CFD numerical analysis has been conducted concerning software packages, solver configuration, numerical models and wind turbines.

Nobile et al. [4] conducted CFD analysis on VAWT and found that different mesh resolution and turbulent model affected the accuracy of the result, but time-step has minimal effect on the result. The result showed that, torque coefficient had increased to 30-50\% after the implementation of omnidirectional stator. Furthermore, the authors stated that VAWT is self-sufficient regardless the wind direction. Cao et al. [5] studied on $2 D$ vertical axis wind turbine using Reynolds averaged Navier stokes equation (RANS) and Realizable $k-\varepsilon$ turbulent model. The result obtained showed that the velocity in the region of wind turbine rotation was much larger that the air flow of the upstream. A larger value of eddy exists in the rear region of the wind turbines blade. The calculated result pointed out in the direction of follow up study.

Wang et al. [6] carried out simulation on $2 D$ vertical axis wind turbine using SIMPLEC algorithm and sliding grid technique in FLUENT. The result showed that, different turbulent model has less effect on velocity field, but relatively has high impact on pressure field with high torque value. Popov et al. [7] presented the methodology and procedure to conduct CFD numerical simulation on a vertical axis wind turbine; Savonius type. The procedure includes; creating geometry, generating mesh, and solver setup. The value of $Y^{+}$criteria at all regimes do not exceed 2.5. The suggested methodology provided a platform to obtain theoretical result. Mohamed et al. [8] investigated Darrieus type wind turbine using Ansys workbench and Gambit meshing tool. Unsteady simulation was performed at different speed ratio based on URANS turbulent calculations. New shapes were introduced in the turbine with higher efficiency compare to other aerofoil by $10 \%$. The result showed that zero pitch angle provided the best performance result.

Based on literature review, the focus of past wind turbine design was mainly to capture the high-speed wind energy. Clearly it is not suitable for low speed wind environment in Malaysia. Therefore, a novel shape of blades is being proposed for an efficient wind energy harvesting turbine relative to low and high-speed wind conditions. This paper discusses on the development and CFD numerical simulation of novel drag type vertical axis wind turbine design. CFD numerical simulation was conducted in two-dimensional due to limitations in computational power. The generated result is studied to find the discrepancy of the result generated at different time-step and mesh resolution. The novel drag type wind turbine design was simulated in FLUENT at static mode and dynamic mode (sliding mesh). 


\section{COMPUTATIONAL METHODOLOGY}

\section{Model Geometry and Domain Configurations}

As illustrated in Figure 1(a), the studied novel drag type vertical axis turbine is composed of three blades and a rotor hub. No specific attention was given to rotor hub and structural support with regards to design. The approach of innovating the wind turbine was inspired by emulating nature properties. Several researchers had conducted and presented research on bio-inspired wind turbine design, which had proved that the design adaptation from nature had increase the credibility in capturing wind energy. Fish et al. [9] and Cognet et al. [10] has thoroughly discussed on bio-inspired wind turbine with respective research. The novelty of the design are the blade curvature and structural bend. The design is inspired from three elements of nature; albatross bird wing shape, pitcher plant and tulips. The hybrid combination of design elements resulted to the geometry shown in Figure 1(a). Modeling of the wind turbine was done in CATIA V5, where the $3 D$ model was dissected by plane sections at blade height of $4 m$ using surface plane splitting technique. Boolean subtract was used to extract the $2 D$ geometry intersecting with the respective plane. Furthermore, the geometry is orientated at different angular position; $0^{\circ}, 45^{\circ}$ and $90^{\circ}$ and the same technique was used for geometry extraction as previously mentioned. In this paper, only $2 D$ model will be simulated and studied due to limitations in computational power. As displayed in Figure 1(b), the turbine consists of three blades, $\mathrm{N}$, with angles of $120^{\circ}$ between each one with a rotor diameter, $D_{r}$, swept area, $S_{A}$, vertical blade height, $H$, hub diameter, $O$, and rotor radius, $R_{r}$. Furthermore, each cavity vane is sweeped at different twist angle, $\alpha$, to generate the curved blade geometry. The rotor diameter of the turbine is $19 \mathrm{~m}$, blade height of $10 \mathrm{~m}$, and hub diameter of $1 \mathrm{~m}$. Aspect ratio, A.R, of 0.53 using Equation 1. Area of the wind turbine is denoted as, $S_{A}$ and has a value of $190 \mathrm{~m}^{2}$ using Equation 2. Since the numerical simulation was conducted in $2 D$, the height, $H$ as shown in Equation 3 are set to $1 \mathrm{~m}$ for reference area configuration. The design was prepared and simulated in megawatt scale in order to study the behavior with regards to the dimensions. Since no predefined scale and dimension benchmark was used in the preparation of the model, reverse engineering study via CFD simulation was conducted in order to find the suitable scale for the wind turbine. In this research, the specific attention was given to cavity vane's geometry in order to analyze the efficiency in wind energy capturing.

$$
\begin{gathered}
A . R=\frac{H}{D_{r}} \\
S_{\text {Actual }}=D_{r} \times H \\
S_{A-\text { simulation }}=D_{r} \times H
\end{gathered}
$$




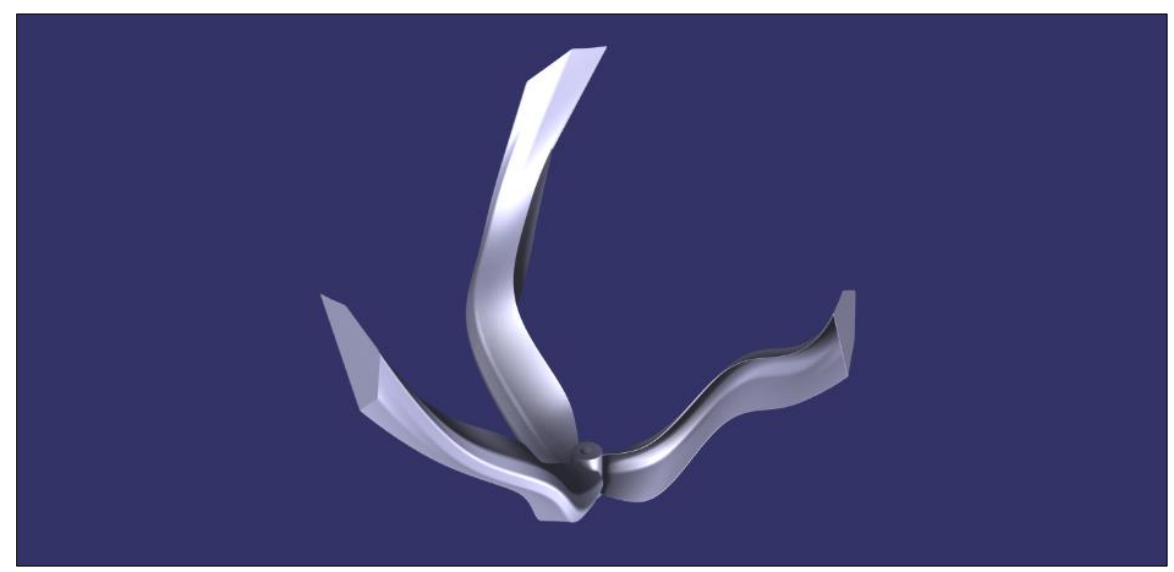

(a)

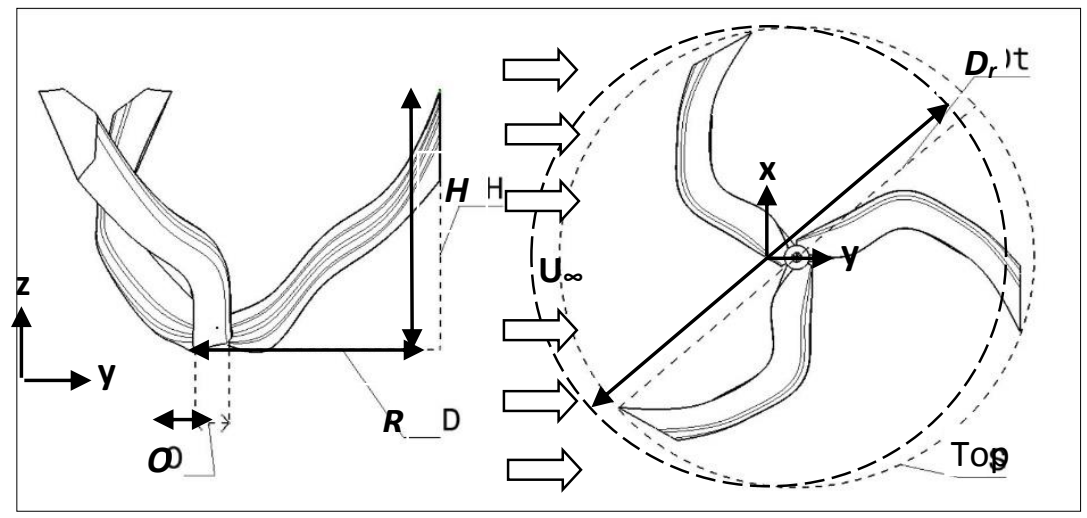

(b)

Figure 1. Wind turbine configuration: (a) Proposed drag driven wind turbine design.

(b) Rotation configuration and geometric parameter.

As displayed in Figure 2, the domain is characterized into two parts; main domain and inner sub-domain. The main domain is the open wind tunnel domain as fixed zone and inner sub-domain is wind turbine blade domain as rotating domain. This paper conducted CFD analysis in $2 D$ to save computational time. The analysis was conducted in two mode; static mode at different angular position, and dynamic mode using sliding mesh technique. As displayed in Figure 2(a), the main domain consists of inlet and outlet and symmetrical surrounding stationary wall. Meanwhile, the main domain was modeled to receive free stream velocity from the left. The walls was set as no slip condition to prevent solid blocking effects [11]. Preliminary CFD analysis study was conducted to find the suitable dimension for main domain without requiring high computational power and to avoid solution discretization error. Domain inlet and outlet was set to four times rotor diameter, $4 D_{r}$. The wind turbine domain was placed $2.5 D_{r}$ from inlet and $7.5 D_{r}$ from outlet. Preliminary study indicated that it is preferable to place outlet boundary condition further away from the inner subdomain to prevent reverse backflow and numerical errors. Meanwhile, the inner subdomain of the proposed wind turbine was set to $1.5 D_{r}$ as displayed in Figure 2(b). In dynamic mode, sub-domain as presented in Figure 2(c), was used for sliding mesh simulation. Main 
domain was maintained for both static and dynamic mode of simulation. Appropriate mesh resolution was chosen to avoid high mesh computational time, thus domain dimension as displayed in Figure 2(a) was chosen to proceed to simulation.

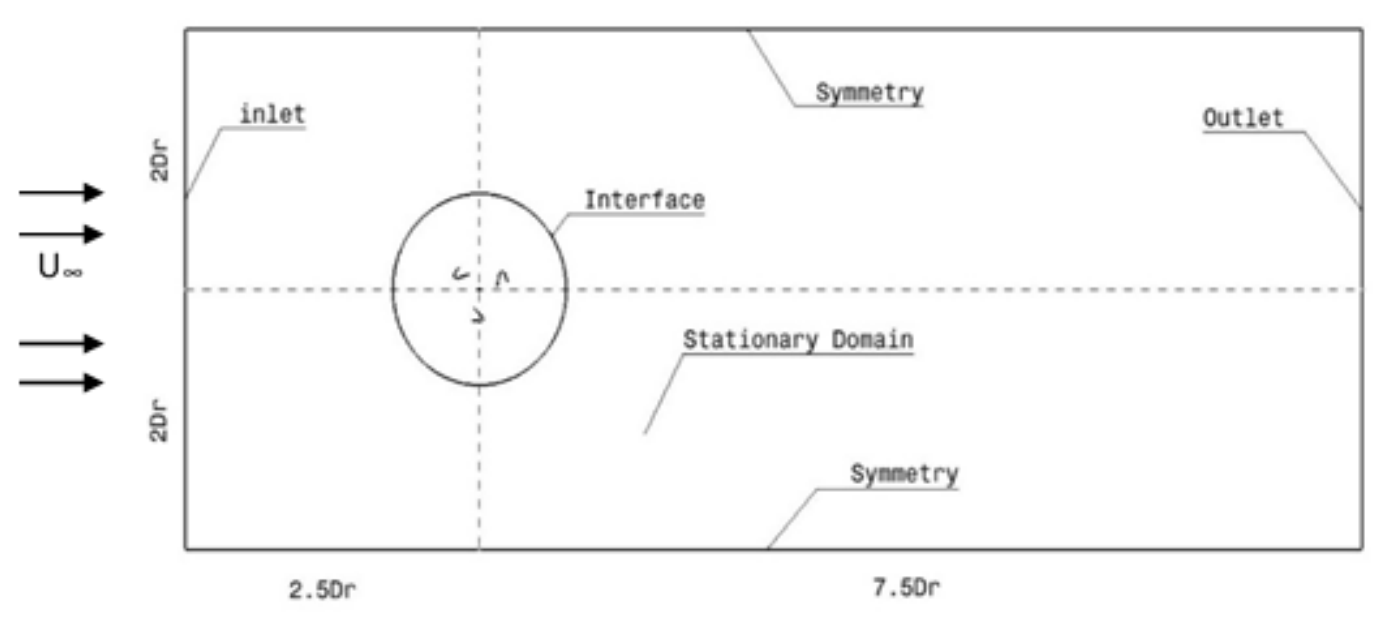

(a)

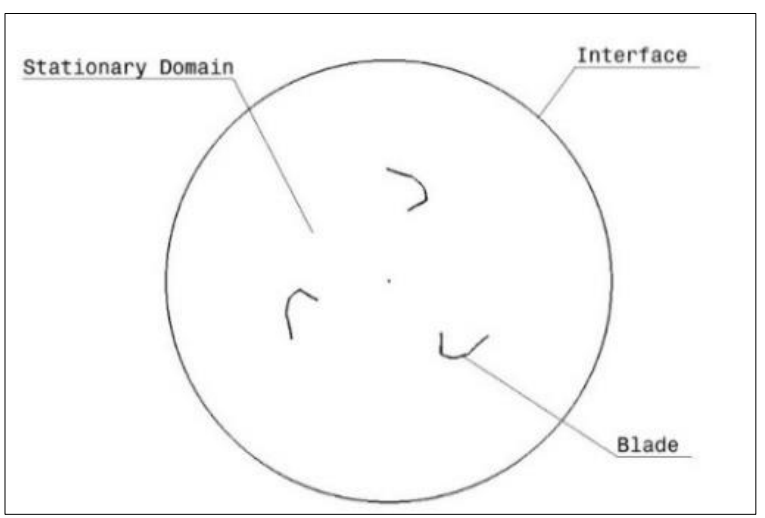

(b)

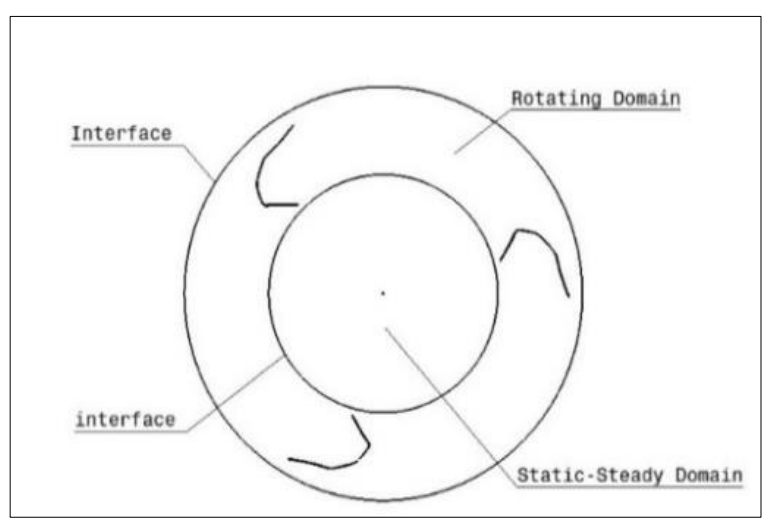

(c)

Figure 2. Domains: (a) Main domain illustration, (b) Sub-domains at elevation of $4 m$ presented turbine's blade for static mesh simulation, (c) Rotating sub domain for sliding mesh technique.

\section{Mesh Topology}

In this research, the mesh modeling is conducted at two different mesh relevance centers; medium and fine to observe the discrepancy in generate result and to conduct mesh dependent study. Due to the complex geometry, spatial discretization under medium resolution with significant difference in nodes lead to poor mesh metrics; orthogonal quality and skewness ratio. Consequently, impact the generated result, causing numerical error and residual instability. Therefore, the relevance centre for medium mesh resolution were set to 40 , in order to increase the orthogonal and skewness quality. Although there is number of nodes and elements are not significant in comparison to fine resolution. The mesh metrics for 
medium mesh is lower than fine mesh. Furthermore, the number of elements and nodes were refined in Fluent via text user interface (TUI) command, therefore increasing the count as reported in Table 1.

Figure 3 illustrates the interfaces of two cell adjacent cell zones in non-conformal mesh configuration. In order to compute the flux across the discretized non-conformal mesh, the interface boundaries surface shared by two cell zone regions must be assigned. Since sliding mesh technique was used in this research, surface shared between rotating sub-domain and fixed main domain was assigned in FLUENT under mesh interface option. Face size or body size is also crucial in interface discretization, large face size and unmatched face size ratio between two domains will lead to mesh overlap and significant interface gaps as displayed in Figure 4(a); 3(b). Appropriate face cell size will save computational time and overlapping interfaces. Connection option in mesh modular will provide a visual indication on the contact region between regions. Although name selection was assigned on the interface regions, additional walls and interior zone adjacent to domain was created. Therefore, interfaces were manually assigned in FLUENT using match option. Match option have coupled the addition walls with regards to the zones, hence created interior zones. Match option works wells for zone regions that do not match well.

As displayed in Figure 5(a), the unstructured computational mesh was generated using a specified mesh generation technique. In main domain structure, all triangular method and body size of $0.2 \mathrm{~m}$ was employed with mesh refinement of 1 . Static mesh sub-domain was mapped using all triangular mesh with face size of $0.1 \mathrm{~m}$ and inflation layers around the blade region. In static mesh simulation, conformal mesh was employed to ensure adjacent nodes between the zones match as displayed in Figure 5(b). In order to create a conformal mesh for static mode simulation, the domain zones were merged into a new singular part zone in order to prevent interfaces issues and to create conformal zones. In static mesh topology, the grid consists of triangular shaped elements in the general area, and triangular mesh in the area close to blade wall with low skewness. The mesh generated under fine mesh setting is 568683 elements and 567698 nodes using conformal mesh technique in static mode. Since the same main domain model was used to analyze moving mesh (sliding mesh technique), interfaces or contact regions between the zone was created in FLUENT and mesh modular. This will ensure zone matching and to prevent empty wall and shadow wall zones to be created. Therefore, non-conformal mesh was used in ensure the separation of moving mesh and stationary mesh as displayed in Figure 5(c). In this research, matching options was used to eradicated additional walls created during modelling and grid generation. 


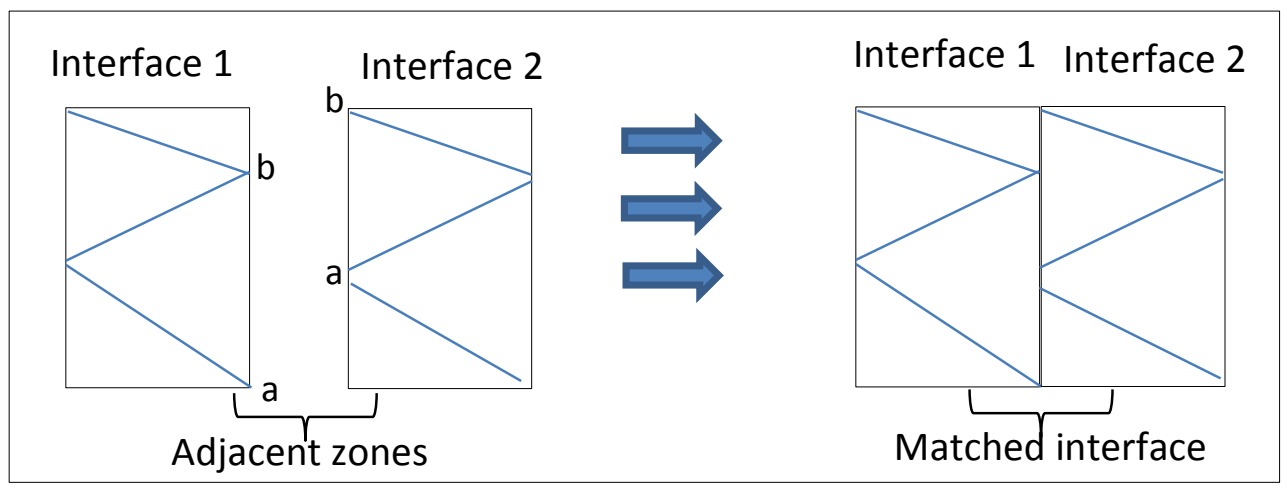

Figure 3. Non-conformal mesh match configuration.

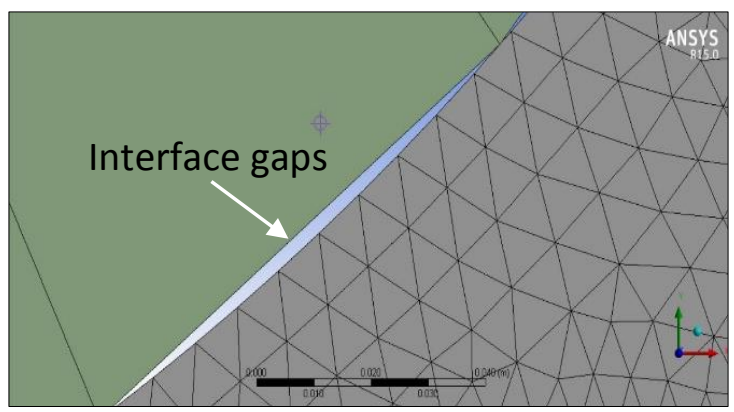

(a)

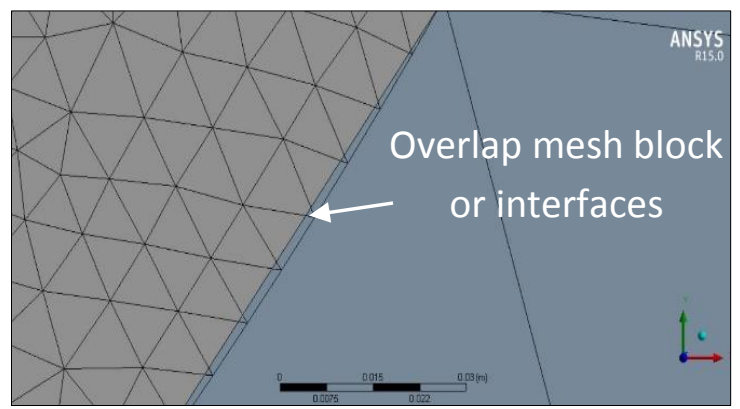

(b)

Figure 4. Mesh interfaces: (a) Mesh interfaces and interior zone visualization, b) interface gaps, (c) Overlapping mesh interfaces.

$Y^{+}$criteria has an impact on the turbulent model performance and the precision of the numerical result [7]. Based on experimental benchmark and theoretical analysis, boundary layer are categorized into 4 groups of $y^{+}$range namely viscous sub-layer $\left(Y^{+} \leq 5\right)$; buffer layer $\left(5<Y^{+} \leq 30\right)$; log law-region $\left(30<Y^{+} \leq 500\right)$ and outer layer [12]. Since SST $k$ - $\omega$ was used in this numerical study, $Y^{+}<1$ are appropriate to capture the flow characteristic at viscous sub-layer. Equation 4-5 are used to estimate the value of first layer thickness wall spacing near the blade wall with approximated desired value of $Y^{+}<1$. In addition, inflation growth rate ratio from the blade surface was set to 1.2 with 17 layers as displayed in Figure 5(d). Furthermore, the skeweness mesh metrics was observed to maintain mesh quality.

$$
\begin{aligned}
Y^{+} & =\frac{\rho u_{f} y}{\mu} \\
u_{f} & =\sqrt{\frac{\tau_{w}}{\rho}}
\end{aligned}
$$

Where $\rho$ is density of air, $u_{f}$ is friction velocity, $y$ denotes the height of layer from the wall, $\mu$ represents dynamic viscosity and $\tau_{w}$ represents shear stress. 


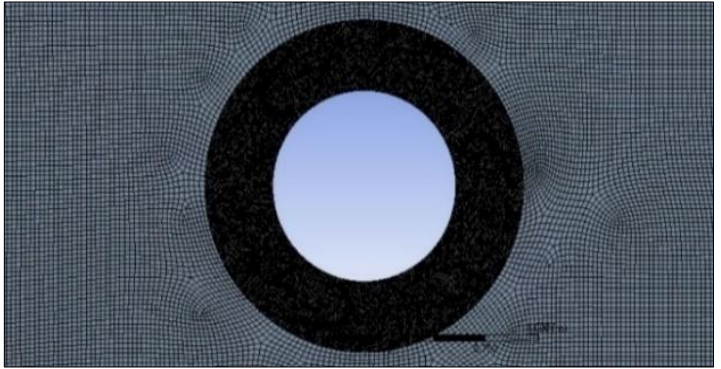

(a)

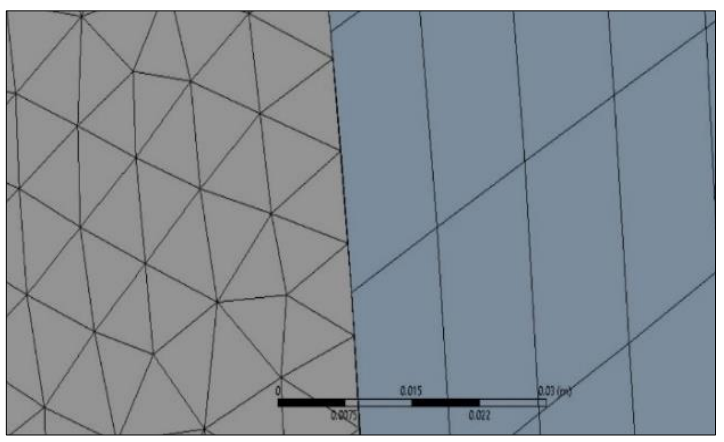

(c)

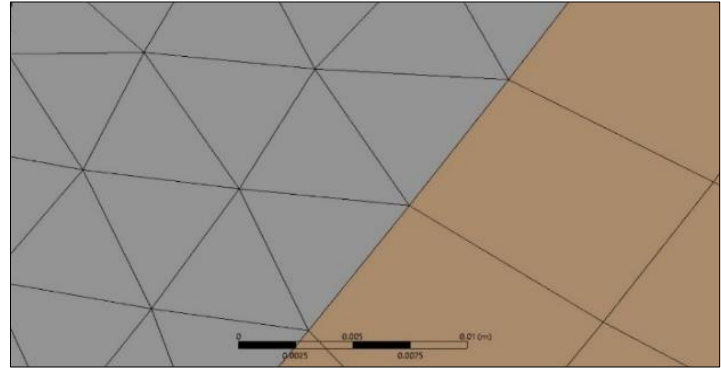

(b)

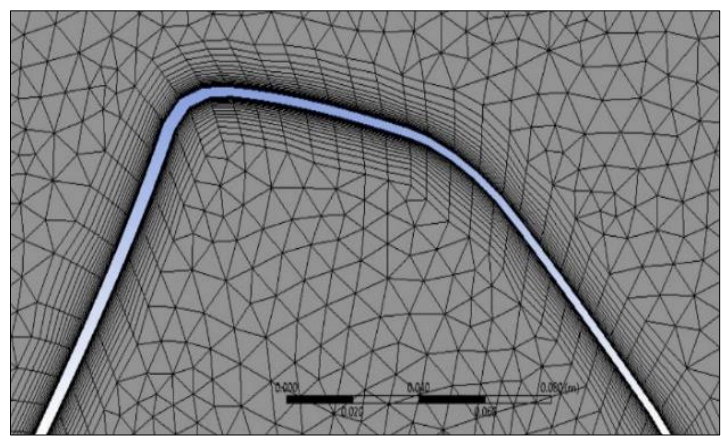

(d)

Figure 5. Mesh configuration: (a) Mesh resolution of static mesh, (b) Conformal mesh, (c) Non-conformal mesh, (d) Inflation layers at blade wall.

Table 1. Mesh Parameters and configurations for static mesh and sliding mesh

\begin{tabular}{ccccc}
\hline Grid type & $\begin{array}{c}\text { Sub } \\
\text { Domain } \\
\text { face size }\end{array}$ & $\begin{array}{c}\text { Inflation } \\
\text { layer }\end{array}$ & $\begin{array}{c}\text { Nodes (conformal } \\
\text { mesh) }\end{array}$ & $\begin{array}{c}\text { Nodes (non-conformal } \\
\text { mesh) }\end{array}$ \\
\hline Medium & $0.1 m$ & 17 & 564042 & 395203 \\
Fine & $0.1 m$ & 17 & 568683 & 395485 \\
\hline
\end{tabular}

\section{Turbulent Modeling}

Turbulent regime or flow type for external flow can be predicted by using Reynolds number equation as illustrated in Equation 6.

$$
R e=\frac{\rho U_{\infty} x}{\mu}
$$

Where $U_{\infty}$ is free stream velocity, $x$ represents the physical length, $\mu$ denotes as dynamic viscosity. In this paper, Equation 6 was used to conduct numerical simulation and to understand the flow type, where rotor diameter is $D_{r}$. Therefore, the Reynolds number of wind turbine region based on Equation 7 is $121.6 \times 10^{5}$, where dynamic viscosity, $\mu$ is 1.849 $\times 10^{-5} \mathrm{~kg} / \mathrm{ms}, \rho$ is $1.184 \mathrm{~kg} / \mathrm{m}^{3}$ at $25^{\circ} \mathrm{C}$ and standard atmospheric pressure. 


$$
R e=\frac{\rho D_{r} U_{\infty}}{\mu}
$$

In this study URANS model was used to analyze complex non-linear result generate Navier stokes equations, by extrapolating Reynolds decompositions as illustrated in Equation 8. In this computational study, $k-\omega$, two-equation turbulent model as illustrated in Equation 9-10 was used because of its robustness and stability in analyzing fluid flow field. Although, $k-\varepsilon$ is more widely used turbulent model [13], SST is more well suited for a stable numerical simulation based on empirical observation for this study. Meanwhile, governing equation are simplified to $x$ and $y$ components for 2D model CFD analysis, as shown in Equation 11-13. Reynolds Decomposition

$$
u(x, y, z, t)=\bar{u}(x, y, z)+u^{\prime}(x, y, z, t)
$$

Turbulent kinetic energy, $k$-equation

$$
\frac{\partial k}{\partial t}+U_{j} \frac{\partial k}{\partial x_{j}}=P_{k}-\beta^{*} k \omega+\frac{\partial}{\partial x_{j}}\left[\left(v+\sigma_{k} \nu_{T}\right) \frac{\partial k}{\partial x_{j}}\right]
$$

Specific rate of dissipation of kinetic energy, $\omega$ - equation

$$
\frac{\partial \omega}{\partial t}+U_{j} \frac{\partial \omega}{\partial x_{j}}=a S^{2}-\beta \omega^{2}+\frac{\partial}{\partial x_{j}}\left[\left(\nu+\sigma_{\omega} \nu_{T}\right) \frac{\partial \omega}{\partial x_{j}}\right]+2\left(1-F_{1}\right) \sigma_{\omega^{2}} \frac{1}{\omega} \frac{\partial k}{\partial x_{i}} \frac{\partial \omega}{\partial x_{i}}
$$

Continuity Equation

$$
\frac{d}{d t} \int_{c v} \rho v+\int_{c s} v \bullet n \rho d A=0
$$

Momentum Equation

$$
\begin{gathered}
\rho\left(\frac{\partial u}{\partial t} u \frac{\partial u}{\partial x}+v \frac{\partial u}{\partial y}\right)=-\frac{\partial P}{\partial x}+\mu\left(\frac{\partial^{2} u}{\partial x^{2}}+\frac{\partial^{2} u}{\partial y^{2}}\right) \\
\rho\left(\frac{\partial v}{\partial t}+u \frac{\partial v}{\partial x}+v \frac{\partial v}{\partial y}\right)=-\frac{\partial P}{\partial y}+\mu\left(\frac{\partial^{2} v}{\partial x^{2}}+\frac{\partial^{2}}{\partial y^{2}}\right)
\end{gathered}
$$

\section{Boundary Condition}

In this research the boundary condition for static mode simulation are as follows; wind velocity at inlet is set to $10 \mathrm{~m} / \mathrm{s}$ in x-axis direction with zero pressure gauge gradient, since URANS model using two-equation turbulent transport model $k$ - $\omega$ was used for computational analysis, turbulent parameter is calculated using Equations from 14-18. Turbulent intensity, $I$, is equal to $2.8 \%$, turbulent kinetic energy, $k$, is $0.1131 \mathrm{~m}^{2} / \mathrm{s}^{2}$, turbulent rate of dissipation of kinetic energy, $\omega$, is $4.44 \mathrm{~s}^{-1}$, hydraulic diameter, $D_{h}$ is $1.97 \mathrm{~m}$, turbulent length scale, $l$, is $0.138 \mathrm{~m}$, and turbulent viscosity ratio, $\mu_{t} / \mu$, is $1.71 \times 10^{3}$. Meanwhile, in outlet is set to pressure outlet where gauge pressure and velocity is zero. As displayed in Figure 2(a), stationary walls are set to symmetry has no boundary condition. 
In the case for dynamic mode, sliding mesh technique was used, where nonconformal mesh technique was employed to create separate interfaces; namely stationary domain and moving mesh domain. This will ensure that the nodes within the boundaries of the domain were not matched or identical. Walls in stationary domain was set to no-slip condition. In sub-domain mesh motion function was employed (sliding mesh technique), where the rotation axis origin $(x, y)$ was set to $(0,0)$, and rotational velocity was set to 40 $R P M$ in the $+z$-direction. Furthermore, wind turbine blade's wall was set to no-slip condition. SST $k$ - $\omega$ model was used to ensure $Y^{+}<1$ to accurately capture flow characteristic at viscous sub-layer and also to study the mesh resolution. In sliding mesh simulation, two sets of simulation were conducted at two inlet velocity values of $10 \mathrm{~m} / \mathrm{s}$ and $25 \mathrm{~m} / \mathrm{s}$ as upper bound and lower bound values while mesh motion $R P M$ value remain constant for both cases.

Turbulent intensity, $I$

$$
I=\frac{u^{\prime}}{\bar{u}}=0.16(R e)^{-\frac{1}{8}}
$$

Turbulent kinetic energy, $k$

$$
k=\frac{3}{2}\left(u_{\infty} I\right)^{2}
$$

Specific rate of dissipation of kinetic energy, $\omega$

$$
\omega=\left(C_{\mu}\right)^{3 / 4} \frac{k^{1 / 2}}{l}
$$

Turbulent length scale, $l$

$$
l=0.07 L
$$

Turbulent viscosity, $\left(\frac{\mu_{t}}{\mu}\right)$

$$
\frac{\mu_{t}}{\mu}=(C \mu) \rho \frac{k^{2}}{\mu \varepsilon}
$$

\section{Solver Configuration}

In this paper, the model is simulated at unsteady state, at different time-step and mesh resolution to find the level of agreement by residual monitoring in lift and drag monitoring for both modes (Static and dynamic mode). In this transient simulation for static mode, the solver type was set to pressure based incompressible flow at absolute velocity formulation. The turbulent model was set to two-equation turbulent transport model, SST $k$ - $\omega$. Turbulent model constants for SST $k-\omega$ are maintained to default values. In order to maintain stability in numerical oscillation, explicit relaxation factor (ERF) momentum and pressure variable values are set to 0.6 and courant number are set to 10 . In the solution methods for pressurevelocity coupling, COUPLE scheme was used, where pressure, momentum, turbulent kinetic, and dissipation of kinetic energy are set to second order upwind. Meanwhile convergence criteria in residual monitor was set to $10^{-5}$ for all residuals. In order to save computational time, the simulations were conducted at 0.0001, 0.0002 and 0.0003 time-steps. Meanwhile in reference value, circular area was set to $14.4 \mathrm{~m}^{2}$ and length was set to rotor radius $7.2 \mathrm{~m}$. This is because in $2 D$ simulation the height of the rotor blade is accounted as $1 \mathrm{~m}$ [14], thus 
the area of the wind turbine is $14.4 \mathrm{~m}^{2}$. Furthermore, adaptive mesh refinement technique was used to refine mesh region with high pressure gradient, where the threshold value is adjusted to ensure a stable numerical study and refined mesh setting. Text user interface (TUI) command was used to repair the meshes, where mesh nodes at boundaries and faces was repaired.

Meanwhile, in dynamic mode sliding mesh technique was employed at different mesh resolution and timestep. In order to numerate suitable timestep Equation 19-21 was used to ensure solution convergence with minimal computational time. Time-step selection in sliding mesh technique are crucial since it determines the number to complete the revolution based on desired parametric value. The number of timestep for a complete revolution can be obtained from 'preview mesh motion' option. Based on observation provided by preview mesh motion option, it indicated 15 number of timestep at 0.1 timestep size for one complete revolution. Calculated value based on Equation 21-23, are $d t, 1.5 \mathrm{~s}$ timestep duration per complete rotation. The desired number of rotations was then multiplied with timestep to obtain the rotation duration, hence a total of $6 s$ for four complete rotation $d t_{4 r e v}$. It is recommended to conduct analysis with more than two revolution in order to remove the boundary condition properties and to extract more stable numerical properties after second revolution.

$$
\begin{gathered}
\omega=2 \pi R P S \\
\omega=2 \pi f=\theta-\theta_{0} / d t \\
d t=2 \pi / \omega
\end{gathered}
$$

Solver configuration mentioned above are finalized after several trial and error to find a suitable solver configuration to conduct CFD analysis. Based on observation, standard, and renormalized group $(R N G) k-\varepsilon$ turbulent transport models are not adequate for this study, because of its unstable numerical oscillation in residual under the similar parametric conditions. Realizable $k-\varepsilon$ model generated fairly stable numerical simulation but for adverse pressure gradient it was noticed to generate relatively high adverse pressure than other transport models result along the blade region, even though the mesh and boundary layer inflation are refined and adjusted respectively. Based on empirical observation, SST $k-\omega$ generated stable numerical analysis with satisfying result.

\section{CFD NUMERICAL RESULTS}

\section{Mesh Resolution Sensitivity Study for Static Mesh Mode}

The purpose of mesh dependence study is to find the suitable mesh setting for a low computational cost simulation setting with accurate and reliable result. In this research two mesh mode was analyzed namely static mesh; and sliding mesh. Dimensionless quantity coefficients such as drag, and moment was studied for static mesh under conformal mesh setting. The simulation was conducted using finite volume method (FVM), where the proposed wind turbine design is studied in a finite volume. The turbine is simulated under free stream velocity $U_{\infty}=25 \mathrm{~m} / \mathrm{s}$ with tip speed ratio, TSR of 1.2 . The case was executed under the parametric value mentioned before are based on estimation, literature survey and preliminary simulation study to ensure turbine performance can be analyzed at optimal state. 
Figure 7 shows drag coefficient result against flow time of blade $1 ; 2 ; 3$ individually under two different mesh resolution; fine and medium mesh at $0^{\circ}$ angular position. The purpose of static analysis is to study of the effect of forces on the blade without angular displacement and angular acceleration. Based on the result, each blade presented different static behavior which dictates the performance of the wind turbine. As presented in the result, there is a good agreement between fine and medium mesh, where the generated numerical result of medium and fine mesh overlaps and converges with regards to flowtime. The converged average drag coefficient $C_{D}$ value for blade 1 is $1.3,0.75$ for blade 2 and 0.85 for blade 3 . As presented in Figure 6(a), blade 3 with the defined angular position of $0^{\circ}$ faced directly on upwind free stream wind flow capturing the wind energy, while blade 1 are opposing to blade 3 , therefore its noticeable for blade 3 to generate high drag value due to high pressure drag generated by the flowing wind. Meanwhile, Figure 8 presented the result of moment coefficients at fine and medium mesh resolution at $0^{\circ}$. The average moment coefficient $C_{m}$ is -0.6 for blade $1,0.47$ for blade 2 and 0.75 for blade 3 .

The result shows that, static moment of blade 3 is the highest due to its polynomial concave shape. Blade 1 indicated negative moment value which may result to performance issue which is common in drag type wind turbines. Meanwhile, Figure 9-10 presents the results at static angular position of $45^{\circ}$. The presented result indicates good agreement between fine and medium mesh. Since the angular position is orientated to $45^{\circ}$, each blade presents different numerical result due to the configuration of the blade facing the upwind. Figure 9 presented result for drag coefficients of blade $1 ; 2 ; 3$. Although there is a slight variation in numerical value in the initial solution initialization, the solution for fine and medium mesh converged with a stable average numerical value for drag and moment coefficients. Drag coefficient at $45^{\circ}$ configuration, blade 1 presents a numerical $1.1 C_{D}, 0.64$ for blade 2, and 1.12 for blade 3. Based on generated graphical result, fine mesh indicated a smoother graphical and numerical transition in comparison to medium mesh. Since the angular position changed the drag coefficient numerical values of blade 1 and blade 2 decreased, and there is a rise in drag coefficient for blade 3. The drop of drag force of blade 1; 2 is due to the drop of pressure and wall shear because of the orientation and angular position of the blade facing the upwind. Figure 10 presented the value for moment coefficients at $45^{\circ}$ angular position. Blade 1 indicated $C_{m}$ value of -0.27 , blade $2 C_{m}$ value is -0.42 , and blade $3 C_{m}$ value is 0.89 . Based on the result, at $45^{\circ}$ angular position presented in rise in $C_{m}$ value for blade 3 . The rise of drag and moment coefficient had increase static torque value of blade 3 in concave orientation. Figure 11 and 12 presents mesh dependence study of blade $1 ; 2 ; 3$ at $90^{\circ}$ angular position at fine and medium mesh resolution. Results on both fine and medium resolution indicated a good agreement with converged result. Fine mesh resolution result presented finer and smother graphical response in drag and moment result in comparison to medium mesh setting. In this angular configuration of $90^{\circ}$ the blades have switch angular position, where blade 1 is facing the upwind as illustrated in Figure 6(c). As manifested in Figure 6(c), the blade 1 in $90^{\circ}$ angular position is facing in the upwind direction therefore rise in moment coefficient is observed. Although blade 3 is in leeward direction wind energy is still captured by blade 3 maintaining in positive moment generation as shown in Figure 12(a). As for blade 2 in downwind direction, moment generation drop to negative values as shown in Figure 12(b).

The presented result shows that, the average drag coefficient, $C_{D}$ for blade 1 is 0.51 , blade 2 is 0.99 , and blade 3 is 1.23 . For moment coefficient result, blade 1 presented an 
average $C_{m}$ value of 0.39 , blade 2 is -0.78 , and blade 3 is 0.81 . Based on the numerical result gathered from $0^{\circ}$ to $90^{\circ}$ as displayed in Figure 6, the orientation of the returning blade and upwind facing blade generate positive and negative moment result respectively. Based on observation, upwind facing blade in polynomial concave configuration generate positive moment result, while returning blade in polynomial convex configuration generated negative moment result. This mesh dependence study indicated that fine mesh setting generated and predicted more accurate flow field properties than medium mesh.

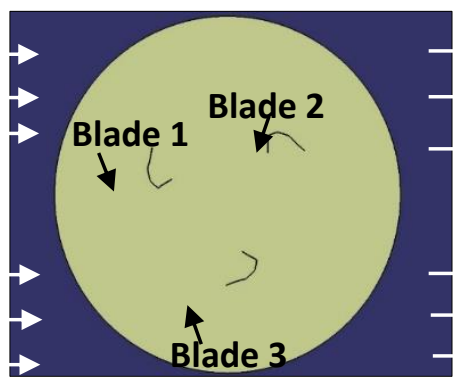

(a)

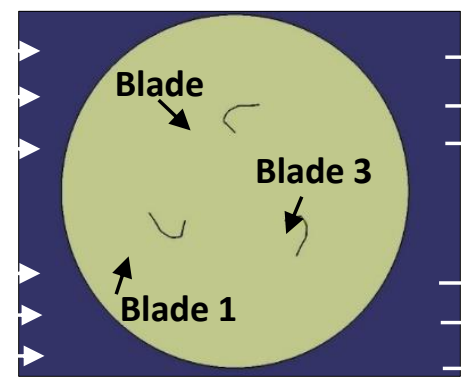

(b)

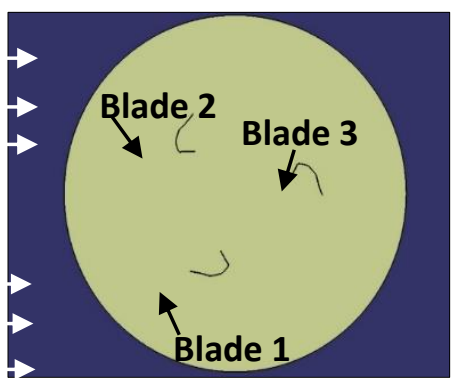

(c)

Figure 6. Blades configuration at different angular position with upwind direction: (a) $0^{\circ}$ angular position, (b) $45^{\circ}$ angular position, (c) $90^{\circ}$ angular position.

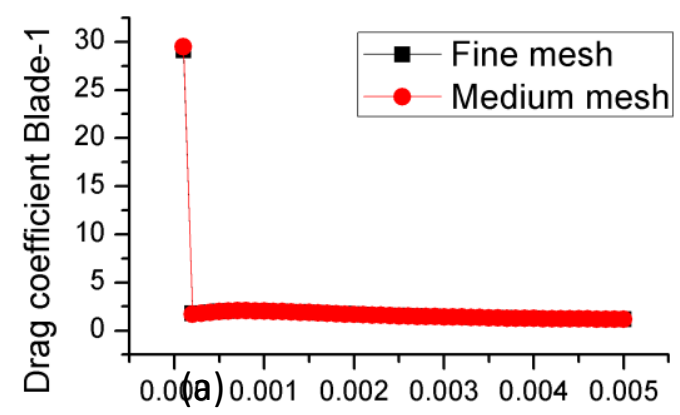

Flow time

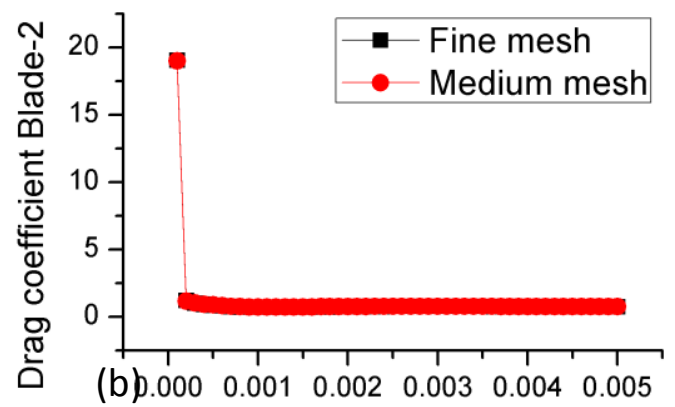

Flow time

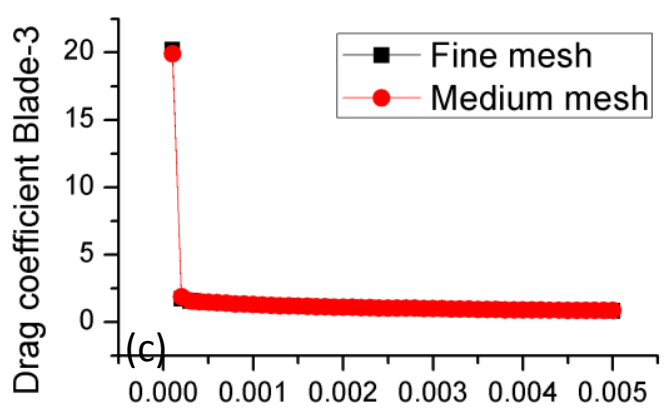

Flow time

Figure 7. Static drag mesh dependence study at $0^{\circ}$ : (a) Drag coefficient blade 1 ,

(b) Drag coefficient blade 2, (c) Drag coefficient blade 3 


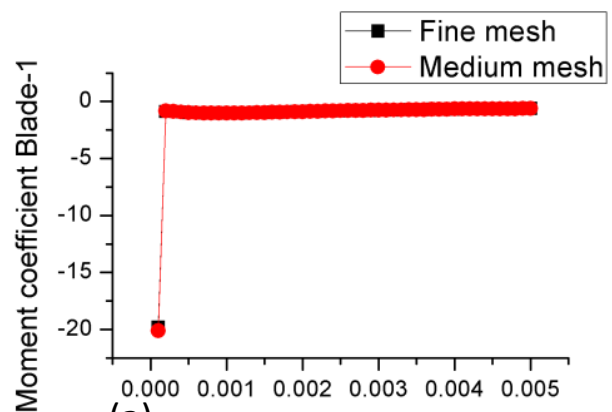

(a)

Flow time

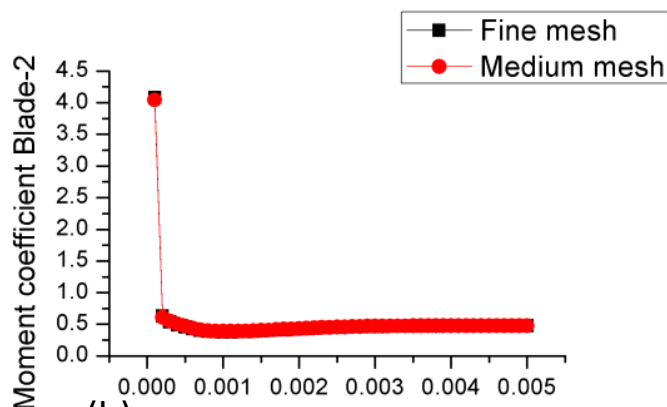

(b)

Flow time

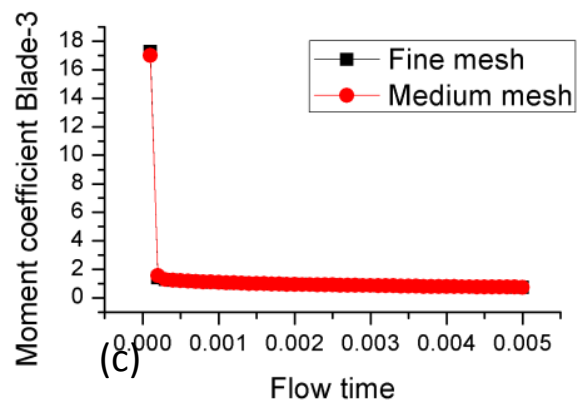

Figure 8. Static moment mesh dependence study at $0^{\circ}$ : (a) Moment coefficient blade 1 , (b) Moment coefficient blade 2, (c) Moment coefficient blade 3

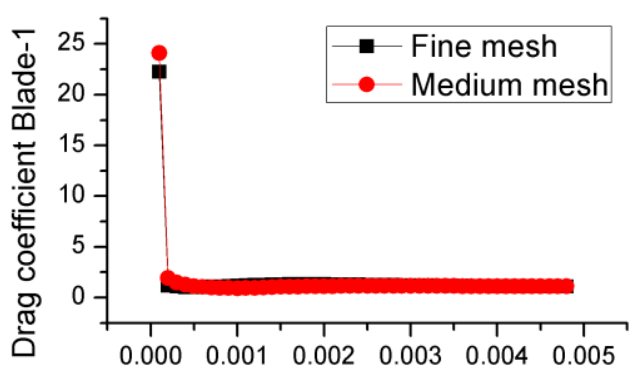

(a)

Flow time

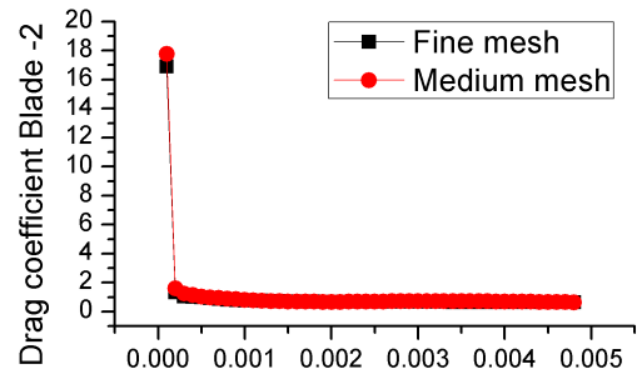

(b)

Flow time

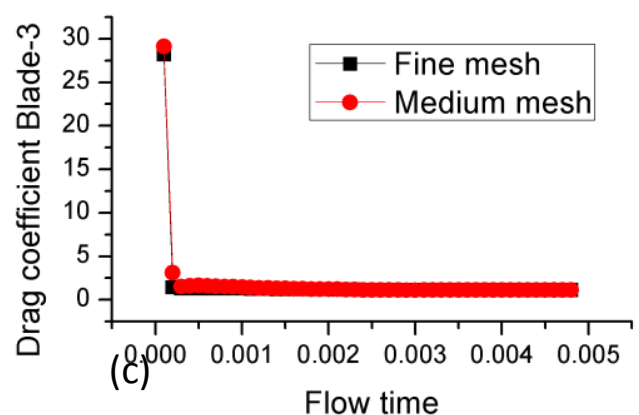

Figure 9. Static drag mesh dependence study at $45^{\circ}$ : (a) Drag coefficient blade 1, (b) Drag coefficient blade 2, (c) Drag coefficient blade 3 

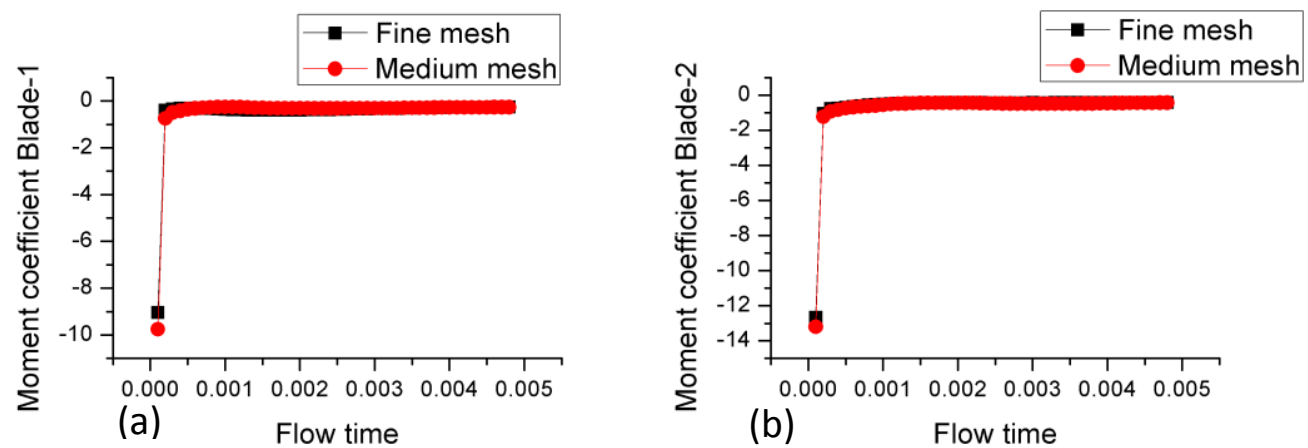

(b)

Flow time

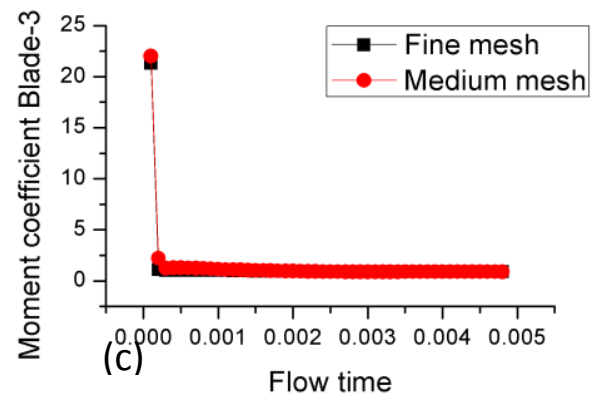

Figure 10. Static moment mesh dependence study at $45^{\circ}$ : (a) Moment coefficient blade 1 , (b) Moment coefficient blade 2, (c) Moment coefficient blade 3

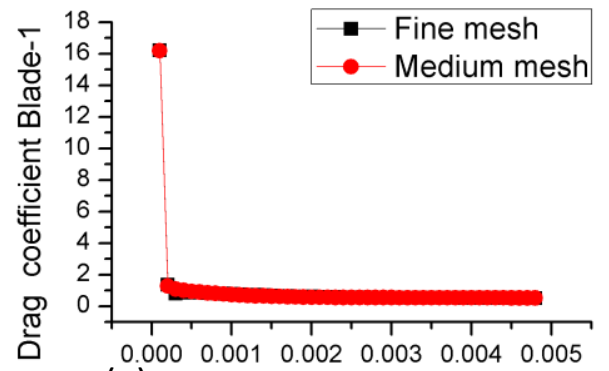

(a)

Flow time

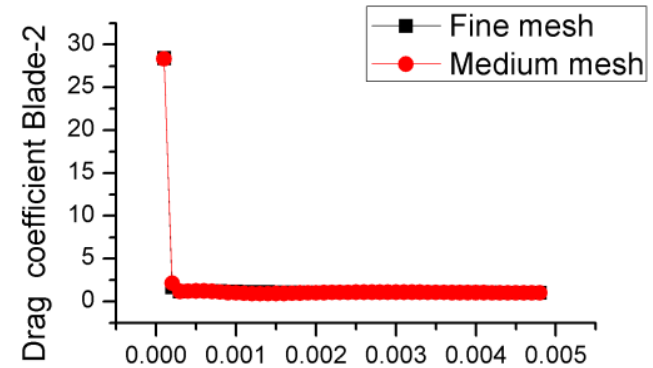

(b)

Flow time

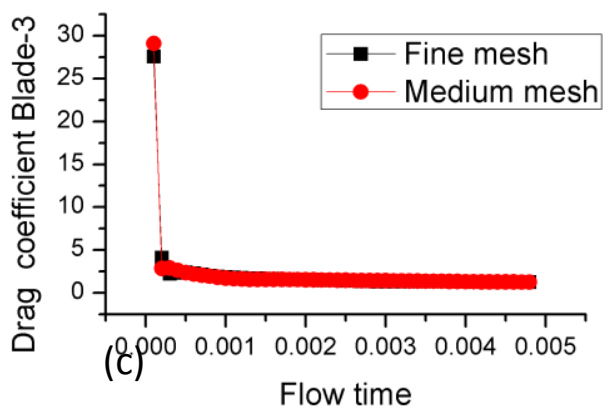

Figure 11. Static drag mesh dependence study at $90^{\circ}$ : (a) Drag coefficient blade 1, (b) Drag coefficient blade 2, (c) Drag coefficient blade 3 


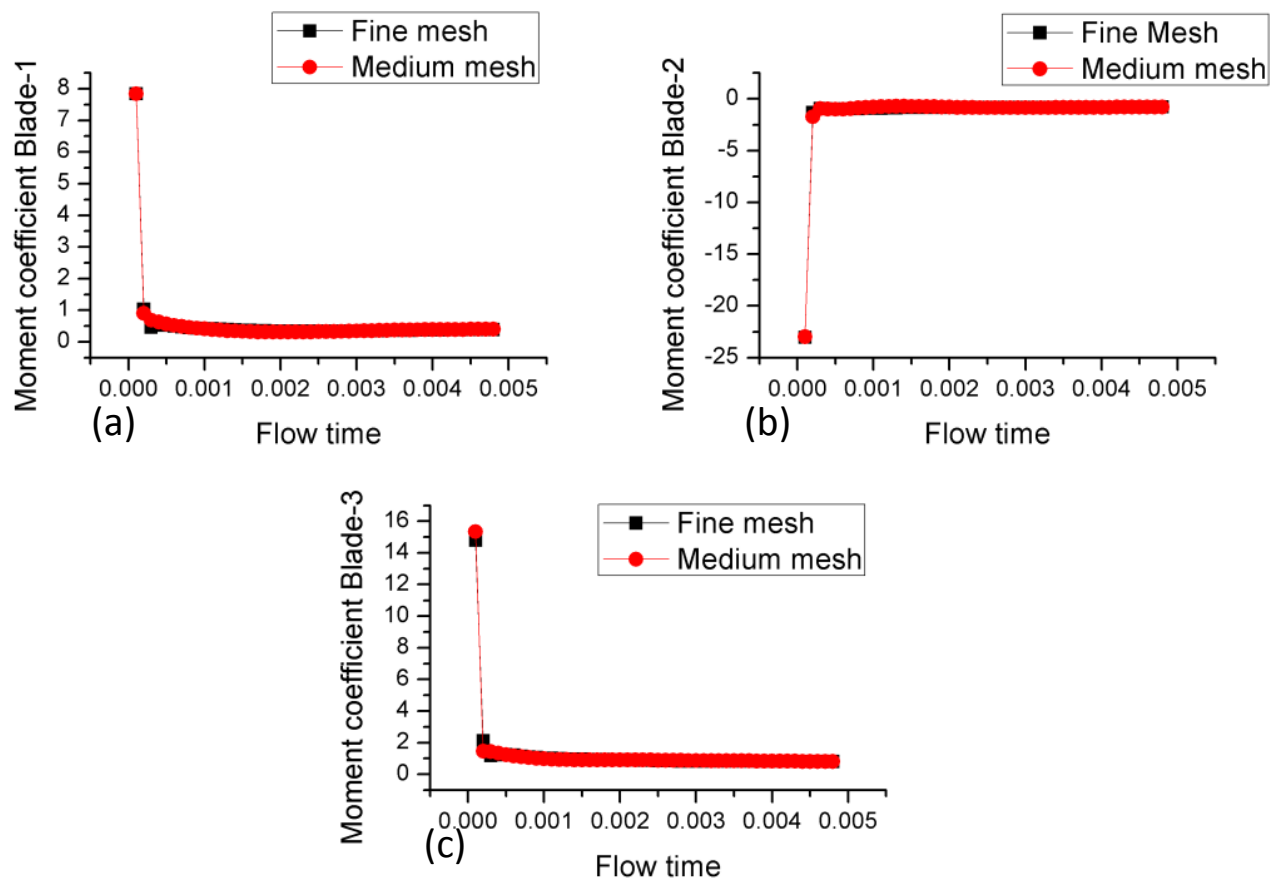

Figure 12. Static Moment mesh dependence study at $90^{\circ}$ : (a) Moment coefficient blade 1, (b) Moment coefficient blade 2, (c) Moment coefficient blade 3

\section{Time step dependence study for static mesh mode}

Time step dependence study is carried out to find the effect of selected time-step size to discretize spatial grid and to generated numerical result. Satrio et al. [15] stated that timestep size and number of time step setting has an impact on the generated simulation result. Selecting the appropriate time-step is crucial in the stability and convergence of the numerical solution. Since the nature of VAWTs are unsteady, appropriate time-step size is required to prevent the processing CPU to incur large computational cost. In this research, three distinguish time-step was selected; $d t=0.0001 ; d t=0.0002 ; d t=0.0003$. In order to save computational time, only blade 1 was selected to conduct time-step sensitivity study as displayed in Figure 13. Based on the presented result, there is a trivial dissimilarity in numerical result in estimating the parameters but shows good convergence in result. It is noticed that, time-step 0.0001 tend to overestimate the drag coefficients in comparison to other time-step, where 0.0003 underestimates the values. Based on the recorded computational time, time-step $d t=0.0002$ is selected because of moderate computational cost in comparison to other discretized time-steps. Although $d t=0.0003$ requires the minimal computational cost and the generated result converges with other timesteps, $d t=0.0002$ provides more stable averaging numerical result, therefore $d t=0.0002$ is chosen. 


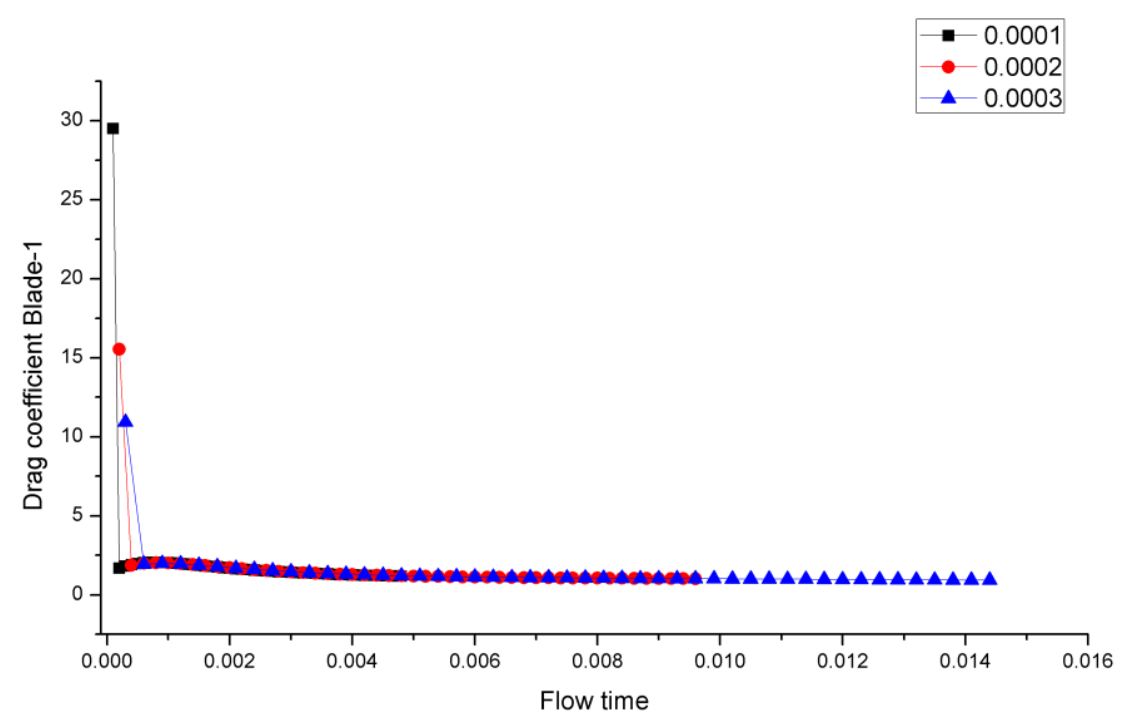

Figure 13. Time-step sensitivity study of blade 1 at $0^{\circ}$.

\section{Mesh resolution sensitivity study for sliding mesh mode.}

Sliding mesh technique is more appropriate in obtaining accurate qualitative data on blades flow field response with regards to unsteady turbulent parameters. In this unsteady simulation, the domains were separated into two zones fixed and rotating zones. The zones are discretized with boundary condition and numerical configuration under non-conformal mesh settings. In order to save computational cost, a preliminary computational time study was conducted on two different rotating zone construction under similar mesh and solver configuration. As displayed in Figure 2, two types of rotating zone were presented, completely constructed zone and hollow constructed zone. As predicted hollow constructed zone requires less meshing and simulation time than completely constructed zone as reported in Table 2. Furthermore, there is no distinct discrepancy in numerical values between the two constructed zones, therefore hollow rotating zone was chosen for simulation study.

In order to validate the sensitivity of discretized grid resolution, mesh dependence study was conducted between fine and medium mesh for each blade individually using sliding mesh technique. Dimensionless quantity such as drag, lift and moment coefficient was analyzed under configured sliding mesh. As displayed in Figure 14-16, fine and medium mesh generated result indicate a trivial dissimilarity in solution. It was observed medium mesh provides sufficient result with given grid resolution as the simulation progresses with regards to iterations, however medium mesh present variation than fine mesh in numerical during the initial stage of the cycle. As displayed in Figure 14-16, fine and medium mesh indicated instability in numerical oscillation before becoming a stable numerical oscillation. It also been observed, fine and medium mesh achieved convergence in several point of simulation time after the $1^{\text {st }}$ revolution as displayed in the figures. Therefore, fine mesh configuration was chosen as solution configuration since there is no significance in solution in comparison to medium. Since it is a drag-based wind turbine, it is sensible for the wind turbine to extract high amount kinetic energy from upwind flowing wind in order to gain momentum and to initiate the rotation, therefore resulting in high parametric value in the initial stage. The periodic oscillation stabilized as the revolution progress. Numerical values 
after $1^{\text {st }}$ revolution should be taken into consideration. In order to remove the boundary condition properties in the initial cycle of the simulation.

Table 2. Different rotating zone construction mesh computation time

\begin{tabular}{cccc}
\hline Rotating construction type & $\begin{array}{c}\text { Mesh } \\
\text { computational time }\end{array}$ & Mesh resolution & Nodes \\
\hline Complete zone & 18 mins & Fine mesh & 548623 \\
Hallow zone & 7 mins & Fine mesh & 395485 \\
\hline
\end{tabular}

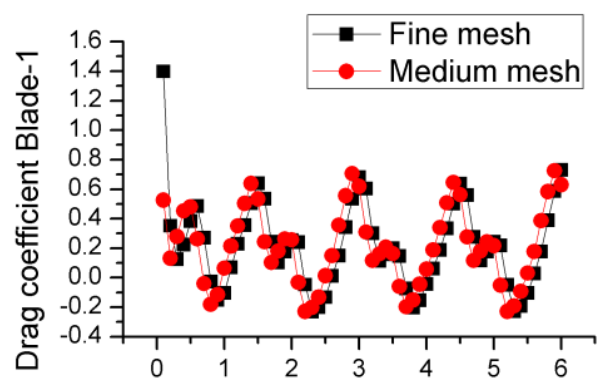

(a)

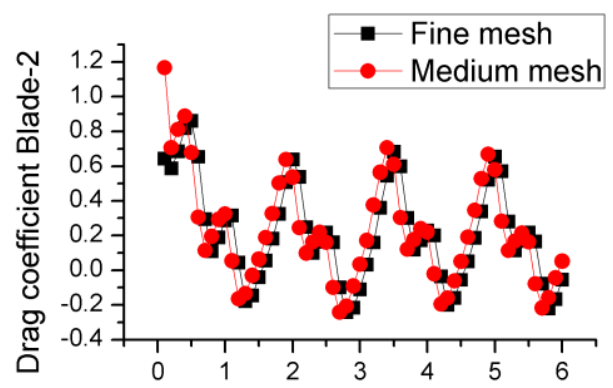

(b)

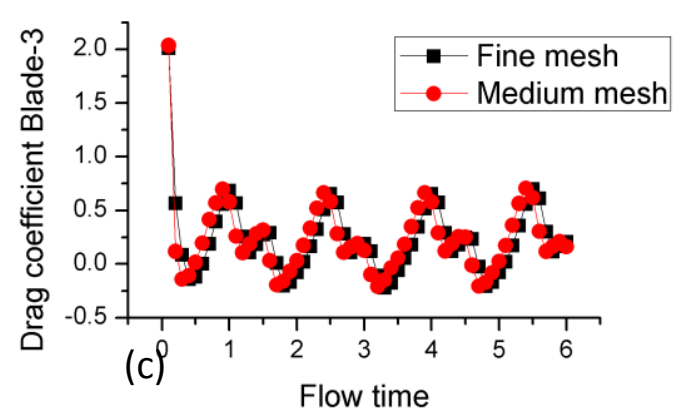

Figure 14. Mesh dependence study of fine and medium mesh: (a) Drag coefficient blade 1, (b) Drag coefficient blade 2, (c) Drag coefficient blade 3. 


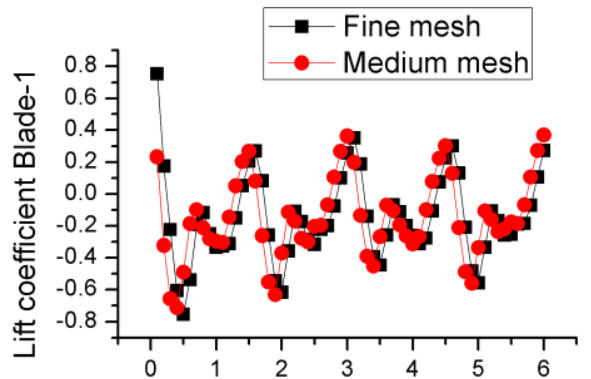

(a)

Flow time

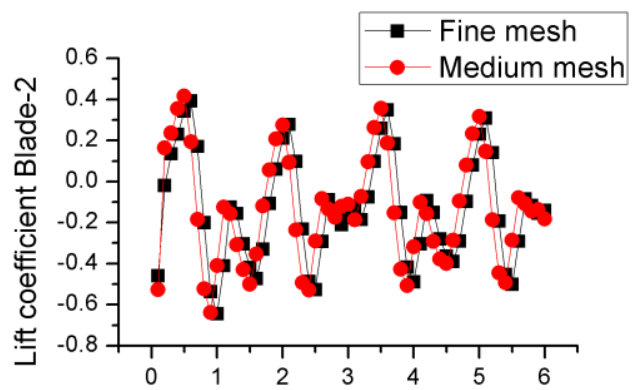

(b)

Flow time

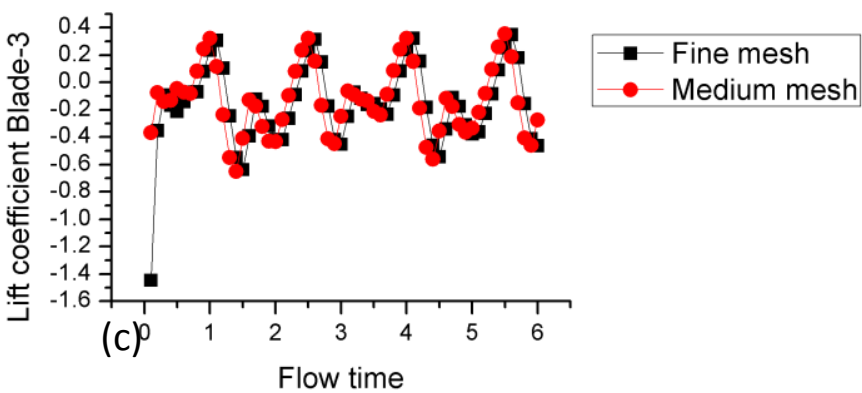

Figure 15. Mesh dependence study of fine and medium mesh: (a) Lift coefficient blade 1, (b) Lift coefficient blade 2, (c) Lift coefficient blade 3.
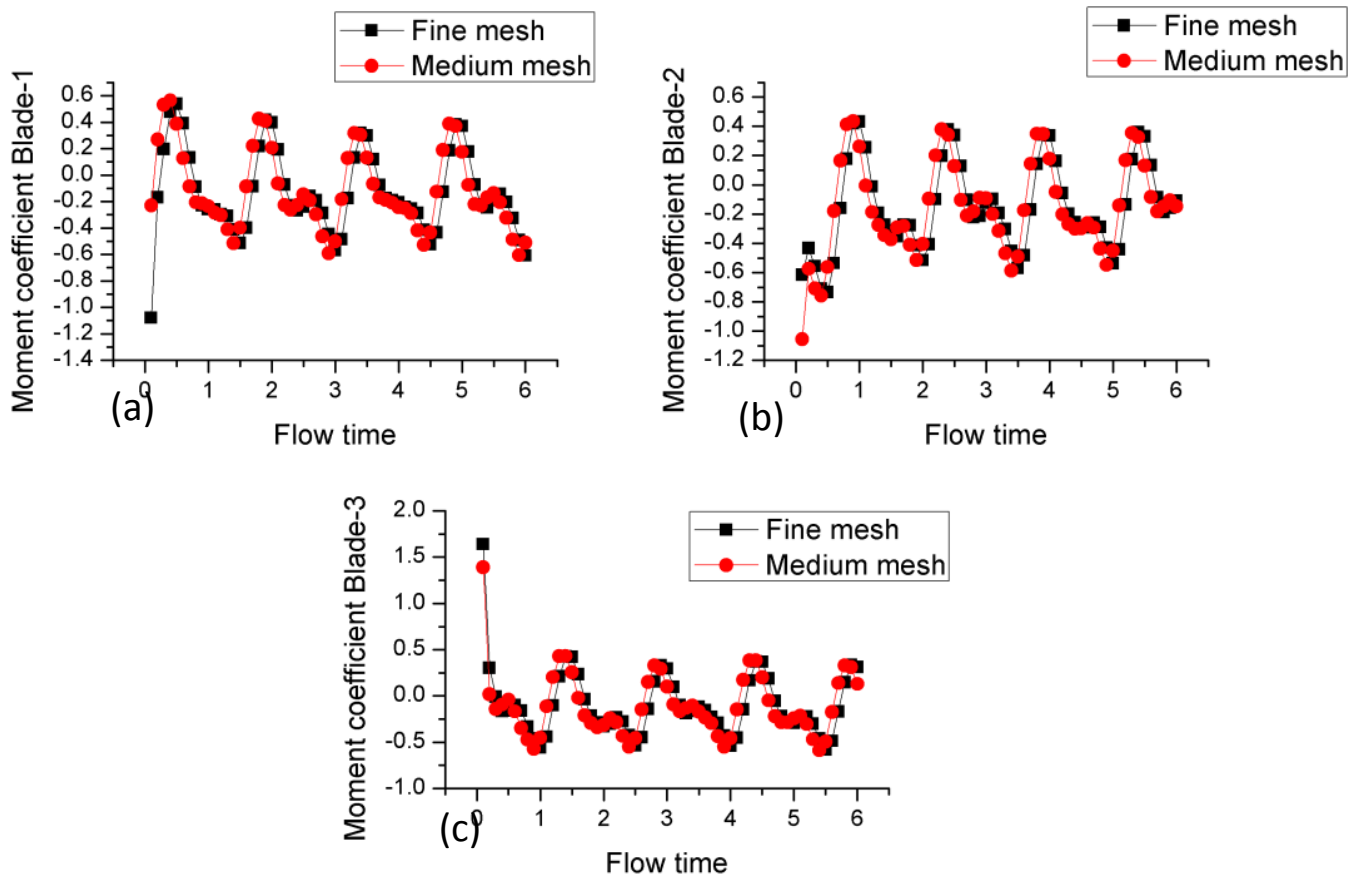

Figure 16. Mesh dependence study of fine and medium mesh: (a) Moment coefficient blade 1, (b) Moment coefficient blade 2, (c) Moment coefficient blade 3. 


\section{CONCLUSION}

This paper presents mesh and time-step sensitivity study of proposed wind turbine design. The objective of the presented mesh and time-step convergence study of is to find the CFD configuration at adequate computational cost for future simulations. The SST $k-\omega$ turbulent transport model in this analysis performed well in capturing the flow field properties in viscous sublayer region as per defined boundary layer thickness in boundary condition configuration. Residual monitoring under SST $k$ - $\omega$ model offered more stable periodic oscillation and fulfilling convergence criteria in comparison to other turbulent transport models. Mesh sensitivity study showed good agreement between fine and medium mesh with trivial dissimilarities in both static and sliding mesh. Although fine mesh resolution consumes slightly higher computational power than medium mesh, fine mesh resolution illustrated higher competency than medium mesh in capturing flow field properties. Static mesh showed good convergence at different time-step size, with trivial dissimilarities between the chosen time-steps. Therefore $d t=0.0002$ was chosen to save computational time. The next step of the research is to study the proposed design with regards to aerodynamic performance and turbine efficiency.

\section{ACKNOWLEDGMENT}

This research work was conducted under Grant number RDU170385. We thank UMP for providing the computing resources. We would like to thank to anonymous reviewers for their helpful comments and suggestion, which helped to improve the quality of the paper.

\section{REFERENCES}

[1] Sun X, Chen Y, Cao Y, Wu G, Zheng Z, Huang D. Research on the aerodynamic characteristics of a lift drag hybrid vertical axis wind turbine. Advances in Mechanical Engineering 2016; 8(1):168781401662934.

[2] Bethi RV, Laws P, Kumar P, Mitra S. Modified Savonius wind turbine for harvesting wind energy from trains moving in tunnels. Renewable Energy 2019; 135:10561063 ,.

[3] Schubel PJ, Crossley RJ. Wind Turbine Blade Design. Energies 2012; 5(9): 34253449

[4] Nobile R, Vahdati M, Barlow JF, Mewburn-Crook A. Unsteady flow simulation of a vertical axis augmented wind turbine: A two-dimensional study. Journal of Wind Engineering and Industrial Aerodynamics 2014; 125:168-179.

[5] Cao L, Wang H, Ji Y, Wang Z, Yuan W. Analysis on the influence of rotational speed to aerodynamic performance of vertical axis wind turbine Procedia Engineering 2012; 31: 245-250.

[6] Wang H, Wang J, Yao J, Yuan W, Cao L. Analysis on the aerodynamic performance of vertical axis wind turbine subjected to the change of wind velocity. Procedia Engineering 2012; 31: 213-219.

[7] Ahmedov GPA, Tujarov K. Methodology for Numerical Modeling the Performance 
of Vertical Axis Wind Turbines. University of Ruse Proceedings, ISBN 1311-332. 53. $194-201$.

[8] Mohamed MH, Ali AM. Hafiz AA. CFD analysis for H-rotor Darrieus turbine as a low speed wind energy converter. Engineering Science and Technology, an International Journal 2015; 18(1): 1-13.

[9] Fish FE, Weber PW, Murray MM, Howle LE. The tubercles on humpback whales' flippers: Application of bio-inspired technology. Integrative and Comparative Biology 2011, 51(1) 203-213.

[10] Cognet V, Courrech Du Pont S, Dobrev I, Massouh F, Thiria B, Bioinspired turbine blades offer new perspectives for wind energy. Proceedings of the Royal Society A: Mathematical, Physical and Engineering Sciences 2017 ; 473(2)198. 20160726.

[11] Nobile R, Vahdati M, Barlow JF, Mewburn-Crook A. Unsteady flow simulation of a vertical axis augmented wind turbine: A two-dimensional study. Journal of Wind Engineering and Industrial Aerodynamics 2014; 125: 168-179.

[12] Lam HF, Peng HY. Study of wake characteristics of a vertical axis wind turbine by two- and three-dimensional computational fluid dynamics simulations. Renewable Energy 2016; 90: 386-398.

[13] Soe TM, Khaing SY. Comparison of Turbulence Models for Computational Fluid Dynamics Simulation of Wind Flow on Cluster of Buildings in Mandalay. International Journal of Scientific and Research Publications 2017; 7(8): 337-350.

[14] Tian W, Song B, VanZwieten J, Pyakurel P. Computational Fluid Dynamics Prediction of a Modified Savonius Wind Turbine with Novel Blade Shapes. Energies $2015 ; 8(8), 7915-7929$.

[15] Satrio D, Utama IKAP, Mukhtasor M. The influence of time step setting on the CFD simulation result of vertical axis tidal current turbine. Journal of Mechanical Engineering and Sciences 2018; 12(1): 3399-3409. 